\title{
L'abstract scientifique anglais - français : contraintes et libertés
}

\section{Elizabeth Crosnier}

\section{(2) OpenEdition}

\section{Journals}

Édition électronique

URL : http://journals.openedition.org/asp/4287

DOI : 10.4000/asp.4287

ISSN : 2108-6354

\section{Éditeur}

Groupe d'étude et de recherche en anglais de spécialité

\section{Édition imprimée}

Date de publication : 1 octobre 1993

Pagination : 177-198

ISSN : 1246-8185

\section{Référence électronique}

Elizabeth Crosnier, «L'abstract scientifique anglais - français : contraintes et libertés », ASp [En ligne], 2 | 1993, mis en ligne le 02 mars 2014, consulté le 10 décembre 2020. URL : http:// journals.openedition.org/asp/4287 ; DOI : https://doi.org/10.4000/asp.4287

Ce document a été généré automatiquement le 10 décembre 2020.

Tous droits réservés 


\title{
L'abstract scientifique anglais - français : contraintes et libertés
}

\author{
Elizabeth Crosnier
}

1 L'abstract scientifique est une forme de discours dont la rédaction semble présenter de nombreuses difficultés. Les chercheurs scientifiques en sont conscients car ils connaissent les règles du jeu; ils savent qu'ils subissent en quelque sorte un examen de passage décisif pour leur carrière. Par conséquent, il est important de le définir, de cerner ses fonctions et l'environnement dans lequel il apparait, et enfin de prendre connaissance des paramètres qui interviennent, puisque tous ces éléments ont des incidences sur le langage que l'auteur va adopter. Dans cet article, les choix terminologiques, syntaxiques et stylistiques opérés par les auteurs dans les abstracts du corpus analysé sont explicités. Nous tentons également quelques interprétations rapides après avoir exposé les éléments contraignants qui peuvent, selon les cas, limiter les options ou même éliminer toute alternative. Nous nous interrogeons également sur l'existence de modèles types $d$ ' abstracts et d'un éventuel style canonique qui enfermerait les auteurs dans un véritable carcan.

2 Cette communication consiste à présenter quelques aspects d'une analyse stylistique comparative du langage appliquée à l'abstract scientifique en anglais et en français dans le domaine de la vision informatique. Ce sont les premiers pas vers l'exploration des textes à partir de données quantitatives, opération réalisée en trois étapes. Dans la première étape, il s'agit de mettre en valeur selon leur taux d'occurrences quelques-unes des données statistiques recueillies après le traitement informatique par le logiciel Alceste, logiciel d'analyse de données textuelles créé par Max Reinert (laboratoire de psychologie de l'université de Toulouse le Mirail). Ces données sont classées par catégories linguistiques au cours de la deuxième étape, pour être enfin interprétées. Grâce à cette étude, la première à exploiter le logiciel, nous pensons pouvoir répondre en partie à la question sur ces notions de contrainte et de liberté.

3 Rappelons que le corpus a été constitué à partir d'abstracts publiés dans des actes de colloques français, anglais et américains récents. Les auteurs ont des origines 
excessivement variées et évoluent dans différents contextes professionnels allant du milieu universitaire au centre de recherche en passant par l'industrie. Tous les textes ont été rédigés dans la langue maternelle.

4 Pour aussi rigoureuses que puissent être les méthodes de saisie et de recueil de données, des erreurs dans les statistiques sont toujours possibles mais nous pensons que le pourcentage est infime et sans grande incidence sur les résultats. Nous tenons à préciser que le terme anglais abstract sera conservé puisqu'il est utilisé par la communauté scientifique internationale et qu'il n'y a aucun mot français équivalent satisfaisant, pas même « résumé ».

\section{Justification du choix de l'abstract}

5 Étant donné le thème de ce colloque, l'abstract semblait être la forme de discours la plus appropriée et il a donc été retenu pour l'expérimentation, ceci pour plusieurs raisons.

6 L'abstract est tout d'abord d'un texte court et cependant fini, complet et qui forme un ensemble homogène, ce qui offre quelques avantages. On peut donc limiter l'analyse à un corpus uniquement constitué d'abstracts, sans atteindre un volume démesuré mais tout en obtenant une richesse et diversité intéressantes grâce à la multiplicité des auteurs et la variété des styles. C'est le cas puisque le corpus considéré ne comprend que 16 pages avec cependant 52 textes en français et 18 pages avec 49 textes en anglais, tous d'auteurs différents venant d'horizons très variés.

7 L'abstract représente une sorte de "passage obligé » pour les chercheurs qui veulent diffuser leurs travaux puisqu'il est pratiquement obligatoire même s'il est vrai qu'on trouve actuellement des articles sans abstract ou bien encore dans certains contextes des abstracts étendus qui font office à la fois d'abstracts et d'articles. On peut supposer d'ailleurs que cette nouvelle contrainte répond à un désir de réduire encore davantage le volume des publications et le temps d'accès à l'information. On verra plus loin que ces paramètres ont déjà leur importance pour l'abstract classique. Celui-ci, tel qu'il apparaît dans notre corpus, reste courant dans le domaine de l'informatique. Grâce à ces deux éléments, l'étude porte sur un échantillon représentatif de par sa variété et sa richesse.

Le troisième critère est lié à l'existence d'un certain nombre de règles : exigences venant de l'extérieur et règles inhérentes au type de texte. Cet aspect est particulièrement intéressant à étudier car il peut engendrer de multiples contraintes.

Enfin le dernier point à prendre en considération concerne l'enjeu de l'abstract, c'est-àdire son acceptabilité et sa validation auprès des éditeurs ou organisateurs de rencontres qui décident de l'avenir de la publication. La qualité du texte est par conséquent déterminante et l'argumentation joue alors un rôle prépondérant.

Pour résumer, nous pouvons dire que la notion de contrainte est toujours présente dans ces quatre éléments. Nous avons donc tenté d'en analyser les incidences sur la langue utilisée et si possible de délimiter sa marge de liberté en tant qu'individu et peut-être aussi en tant que représentant d'une certaine culture, française ou anglo-saxonne. C'est pourquoi il nous a semblé intéressant de travailler sur le français et l'anglais, en considérant les aspects linguistiques contraignants et le fonctionnement des deux langues dans un discours bien déterminé. 
11 Nous avons posé l'hypothèse que l'auteur d'abstract doit faire des choix et procéder à des aménagements linguistiques en fonction des contraintes citées, certaines étant impératives, d'autres peut-être moins strictes. Peut-on dire par exemple qu'il y a un style canonique propre à l'abstract? L'auteur peut-il personnaliser son texte, évoluer librement à l'intérieur d'un cadre préétabli, ou bien au contraire est-il enfermé dans un carcan? C'est ce que nous allons essayer de vérifier au cours de l'analyse du langage de l'abstract en français et en anglais à partir du corpus. Mais rappelons d'abord les paramètres qui interviennent dans la rédaction d'un abstract.

\section{Contraintes}

12 Nous allons examiner rapidement les contraintes qui contribuent, plus ou moins, à réduire l'espace de liberté de l'auteur et à motiver ses choix linguistiques.

13 La première des contraintes externes est liée à l'espace occupé par l'abstract et aux conditions de publication, quel que soit le support : papier, disquette, microfiche. Celle-ci se concrétise par un nombre limité de mots, dont l'un des objectifs est de réduire le temps de lecture, donc l'accès à l'information. L'auteur est par conséquent obligé de faire preuve de concision et de choisir judicieusement son vocabulaire.

La deuxième contrainte imposée est ce qu'on peut appeler le "style maison », c'est-à-dire que dans certains cas, une liste de notes, un « guide de bon usage » en quelque sorte, sera envoyé à l'auteur, avec toutes les consignes concernant l'usage de la langue. Les réviseurs, éditeurs et organisateurs de colloques sont les juges en la matière.

Parmi les exigences, on peut noter des remarques concernant l'utilisation du pronom we, la présence de that dans les propositions complétives, la forme active plutôt que la forme passive, voire des listes de mots à éviter. Ce ne sont que quelques exemples, mais les consignes peuvent être nombreuses et variées, portant aussi bien sur les temps, le vocabulaire abstrait, la syntaxe, l'articulation du discours, etc. Ces remarques valables pour tout l'article le sont d'autant plus pour l'abstract. Toutefois les auteurs ne sont pas tous soumis à si rude épreuve ; certains ne reçoivent que quelques consignes relatives à la qualité et l'usage de la langue, voire uniquement à la clarté du message. Les informations obtenues à ce propos émanent toutes des pays anglo-saxons. Aucun document français ne nous est parvenu. Nous pouvons cependant supposer que les chercheurs français sont largement influencés par les Anglophones.

Un troisième élément doit être mentionné : il semble que ces contraintes varient selon les contextes et aussi les disciplines. Les informaticiens paraissent moins exigeants que les mathématiciens ou les spécialistes de mécanique, ce que nous avons vérifié dans notre enquête menée à propos des évaluations faites par les réviseurs.

17 Enfin un paramètre d'une importance capitale intervient au chapitre des contraintes externes, il s'agit des changements : changements d'habitudes et d'attentes, des effets de mode et de culture ainsi que de l'évolution de la science et des langues. Il faut dire que le temps et l'aspect financier jouent des rôles de plus en plus importants, que la tendance est de faire court et concis, de lire vite avec un maximum d'efficacité. On a aussi remarqué en général un goût plus prononcé pour des phrases de moins en moins complexes et plus courtes. Ceci est également valable pour le langage scientifique. Kocourek notait une moyenne de 28-29 mots par phrase (Kocourek 1982), alors que dans notre corpus, la moyenne ne dépasserait probablement pas 20 mots. Cet aspect nous fera 
prendre conscience de la validité toute relative des analyses faites à un moment donné. En effet, il est essentiel d'insister sur le profil fragile et non permanent de l'abstract.

Les autres contraintes ayant des effets sur la langue sont internes c'est-à-dire inhérentes à l'abstract lui-même ; elles sont liées à sa nature et ses fonctions, reconnues ou perçues comme telles par l'auteur indépendamment des consignes qu'il a pu recevoir. On peut vérifier au travers de l'analyse le rôle qui est en général attribué à cette forme de texte ou bien au contraire les écarts par rapport à quelque chose qui ressemblerait à une ligne de conduite. Ces fonctions, qu'elles soient entièrement remplies, ou partiellement, vont obliger l'auteur à aménager le langage, à l'adapter, à choisir ou éliminer. C'est ce que nous allons essayer de démontrer maintenant.

\section{Analyse du corpus}

Avant de commencer l'analyse des textes, rappelons quelques chiffres (voir tableau 1).

Tableau 1

\begin{tabular}{|l|l|l|}
\hline & français & anglais \\
\hline TITRES (cumuls) & 587 & 462 \\
Moyenne mots/titre & 11,2 & 9,4 \\
Nombre de mots minimum & 5 & 3 \\
Nombre de mots maximum & 23 & 19 \\
Phrase la plus longue & 49 & 49 \\
\hline ABSTRACTS & 52 & 49 \\
Nombre de mots (cumuls) & 5983 & 6964 \\
Moyenne mots/abstract & 115 & 142 \\
Nombre de mots minimum & 38 & 39 \\
Nombre de mots maximum & 228 & 387 \\
\hline
\end{tabular}

Même si ce n'est pas le propos essentiel de cette étude, quelques remarques sur les titres peuvent s'avérer utiles. La longueur des titres varie, allant de 5 à 23 mots en français et de 3 à 19 en anglais, les extrêmes étant toutefois des exceptions. Ces titres se composent en général d'une seule phrase fortement nominalisée sans syntaxe. Ils sont très étoffés et comprennent plusieurs adjectifs, compléments de noms et d'attribution. L'anglais remplace souvent les prépositions par des concaténations pouvant atteindre 6 mots. Seuls 2 titres anglais n'en comportent pas. La préposition de est reprise 90 fois, of seulement 10 fois et for 17 fois.

Quelles que soient la structure et les ressources dont disposent les 2 langues, le titre s'avère en général très informatif et explicatif. Le nombre élevé de mots chargés sémantiquement, de marqueurs tels que en vue de, par, à partir de, pour ou le signe de ponctuation < : > 12 fois utilisés en français, 3 fois en anglais, from, ou les formes comme based, using, les gérondifs (17 occurrences en anglais) traduisent le désir de l'auteur d'informer non seulement sur le sujet, mais aussi sur l'expérimentation, la méthode 
utilisée, l'application et l'objectif à atteindre avec même un développement explicatif. En revanche les notions de problèmes et difficultés sont absentes, ainsi que les résultats. Un seul titre français indique: "explication et résultats». Notons également le faible taux d'occurrences des sigles qui sont d'ailleurs plus fréquents en anglais mais non explicités. D'après les consignes fournies aux auteurs, il est souhaitable qu'ils n'apparaissent ni dans le titre ni dans l'article à l'exception toutefois des acronymes, utilisés comme noms de programmes par exemple. Contrairement aux abstracts de mathématiques dont plusieurs titres commencent par on en anglais et sur en français, ce qui rappelle le début des titres latins de..., nous n'en avons trouvé aucun en informatique.

Pour conclure, nous pouvons déjà remarquer des constantes, à savoir :

- la densité et l'étoffement dus à une masse d'informations en un minimum de place, sans syntaxe et formalisée avec concision, surtout en anglais grâce aux concaténations ;

- des mots clés appartenant au vocabulaire spécialisé indiquant déjà avec précision les notions et les opérations accomplies ainsi que les objectifs ;

- des contraintes notoires mettant en valeur le rôle du titre : il est concis mais informatif et permet un accès rapide à l'information.

Ce n'est donc pas surprenant de constater que les abstracts, à l'exception de 6 en anglais et 11 en français, reprennent quelques mots clés, voire tous les mots du titre dans leur première phrase. Il semble que ces contraintes puissent être transposées au niveau des abstracts.

Trois points linguistiques où les notions de contraintes et libertés semblent particulièrement présentes seront abordés dans cet article. Notre choix s'est porté sur la phraséologie de l'abstract dans l'expression des fonctions, l'articulation du discours et enfin l'argumentation. Nous avons volontairement exclu de l'analyse la terminologie qui a déjà donné lieu à de nombreux travaux. Nous nous consacrerons essentiellement à une étude stylistique selon l'orientation adoptée par les spécialistes de traduction (GuilleminFlescher 1981). C'est aussi dans cette optique que nous tenterons de décrypter quelquesuns des messages qui traduisent l'expression d'un style personnel et les différences culturelles puisque nous traitons en parallèle l'anglais et le français. Ce point ne pourra cependant pas être développé dans cet article.

La présentation qui va suivre a pour point de départ les résultats statistiques obtenus grâce au programme Alceste. Son fonctionnement serait trop long à expliquer. Il faut savoir toutefois qu'il est fondé sur le calcul des occurrences, puis sur le classement par catégories grammaticales pour le français, notifiant aussi les formes fléchies, ensuite sur la division par classes des unités de contexte et enfin sur les occurrences d'associations de mots par paires qu'on appelle couples ou de plusieurs mots qu'on appelle segments répétés. C'est à partir de ces données qu'il est possible d'analyser le langage et de tenter quelques interprétations. Le programme fonctionne aussi en anglais mais la version actuelle ne permet pas encore tous les classements, surtout ceux des catégories grammaticales. La prochaine version devrait être complète.

\subsection{Phraséologie/fonctions}

Nous avons constaté un phénomène de récurrences à travers la présence de constructions figées dans les abstracts en général. Nous avons voulu vérifier si cela était vrai dans le domaine de la vision informatique. Le calcul informatisé des occurrences a largement simplifié la tâche. L'abstract constitue un cadre, explorons son contenu. 

notions et des opérations accomplies, on retrouve la même panoplie de mots, combinaisons de formes nominales et verbales aux taux d'occurrences élevés avec les équivalents dans les deux langues. Citons les plus usuelles : décrire le système, l'image, la forme, l'objet; développer; analyse, segmentation, application, implémentation, représentation, vision; et les traductions en anglais avec do, develop, operate, perform.

31 3.1.3. La notion de méthode et de traitement est traduite de la même manière dans les deux langues avec cependant un usage plus intensif en anglais qu'en français du mot technique, plus concret que notre méthode. On trouve également processus, approche et procédure; process et procedure souvent interchangeables chez les informaticiens. Ces mots sont toujours combinés avec les mêmes verbes présenter, use, utilize...

3.1.4. La notion de problème est peu formalisée et se limite en français à des formules combinant problème, résoudre et solution, et en anglais solve, problem, difficulty, issue...

33 3.1.5. La notion de résultats n'apparaît que dans la moitié des textes et elle n'est pas toujours formalisée. La phraséologie est alors limitée en fréquence et en expressions avec essentiellement une formule en français combinant présenter, résultats et obtenir : «Nous présentons des résultats obtenus... Quelques résultats obtenus... sont présentés... » tandis que les Anglophones ne mentionnent que results : "several results are presented... ", ce qui traduit une légère tendance du français à étoffer.

3.1.1. La première formule qui frappe immédiatement exprime la présentation du sujet. Elle se traduit par diverses combinaisons comprenant le verbe présenter avec ses variantes apparaissant dans des phrases assertions essentiellement au présent de l'indicatif, parfois au passé composé, exceptionnellement au futur, et dans des structures toujours actives en français, actives ou passives en anglais :

cet article présente..., ce papier présente..., nous présentons dans cet article....... is presented in this paper

le remplacent tels que proposer, traiter, développer, exposer, avec les équivalents anglais introduce, propose, develop, "...is introduced and implemented. In this paper, a new approach has been proposed... ", avec un total de 111 occurrences en français et 100 en anglais. Le mot article revient régulièrement dans les deux langues. Le français le remplace 5 fois par le marqueur ici. À ce propos, notons l'influence de l'anglais sur le français par l'utilisation du mot papier. L'usage du pronom nous s'est généralisé en français, l'équivalent anglais we est moins usité mais il apparaît de plus en plus fréquemment dans les abstracts actuels. En revanche, le sujet inanimé paper du verbe animé est plus fréquent en anglais. Remarquons ici l'influence réciproque qui s'accroît régulièrement: we est maintenant accepté dans lécrit scientifique, même parfois souhaité, et la structure anglaise imprègne la phrase française. Notons une seule occurrence de on présente. Pour terminer, notons que les
formules de présentation ne sont pas nécessairement en première phrase, et ceci dans les deux langues.

3.1.6. La dernière fonction repérée concerne l'explicitation qui se traduit surtout par based utilisé 40 fois et sa traduction littérale basée qui apparaît 27 fois. La phraséologie est 
réduite. En revanche chaque langue possède d'autres ressources linguistiques ou métalinguistiques :

- ou et l'équivalent or pour désigner une même chose, pratique non généralisée mais courante ;

- notamment, c'est-à-dire, en effet et en anglais that is, namely, such as, called, for example ; avec un taux d'occurrences très faible ;

- un codage tel que les parenthèses qui sous-tendent une pratique de lecture à deux vitesses fréquente, et appréciable, dans le domaine des sciences, utilisées souvent pour les sigles et leurs explications ; cette pratique est adoptée par la communauté scientifique en général.

- les structures appositives qui peuvent définir ou identifier les éléments qui précèdent par souci d'expliciter. Ce processus est rapide et économique en nombre de mots, il évite la lourdeur de phrases complexes et le risque d'ambiguïtés ; il est largement employé dans les deux langues.

- les < : > sont plus fréquents en français.

- l'abréviation «i.e. » est apparue une fois dans chaque langue.

On constate donc en comparant les textes que les auteurs intègrent souvent des éléments explicatifs mais sans étoffement important de la part des Français. Cette notion confirme la nécessité de désambiguïser l'énoncé mais avec économie et concision.

Comme remarques globales sur la phraséologie, termes et combinaisons utilisés de manière intensive pour exprimer les fonctions essentielles, remarquons tout d'abord:

- une grande similitude entre les deux langues avec toutefois quelques variantes sur les taux d'occurrences ainsi que quelques traces de métissage, surtout de l'anglais vers le français, ce qui engendre parfois des expressions étranges calquées sur l'anglais : "La méthode développée autorise l'utilisation... ».

- <\#ITALIQUES\#></\#ITALIQUES\#>Ensuite un nombre important de variations sur un même thème à partir de mots sémantiquement vides (deadwood) qui, associés aux termes techniques les plus fréquents comme algorithme, texture, graphe, etc., se chargent de sens dans le contexte donné.

- Un taux d'occurrences élevé pour un nombre de formes restreint : on retrouve toujours les mêmes termes répétés plusieurs fois si nécessaire dans le même texte.

- On remarque que dans les constructions françaises, les combinaisons qui incluent permettre sont particulièrement prisées par les scientifiques. On note aussi 71 occurrences de use et utilize que les Anglophones intègrent volontiers.

- La structure la plus communément utilisée dans ces formules est la forme passive qui devient naturelle chez les scientifiques français: "un processus est alors présenté... ». En revanche, certains tiennent à garder le rythme de la phrase française. Avec la prédominance du temps présent et de la forme passive dans des phrases déclaratives, c'est ce qui constitue les traits syntaxiques les plus notoires.

La dichotomie traditionnelle et si fortement ancrée à propos de l'anglais, langue concrète et dynamique, et le français plus abstrait et statique, ne semble pas tellement justifiée dans la traduction des fonctions sous forme de blocs " prêts à l'emploi ». Les deux langues utilisent dans l'ensemble les mêmes termes et les mêmes notions abstraites quand cela est nécessaire. Cette phraséologie est excessivement présente dans les deux langues et laisse peu de place à l'originalité. On pourrait d'ailleurs discuter de l'utilité de certains éléments. emprisonné dans cette phraséologie contraignante ou a-t-il possibilité de manifester un 
style plus personnel? Nous répondrons que les différentes étapes qui composent son message de présentation des fonctions lui laissent peu de libertés. Il peut éventuellement remplacer ces mots vides par d'autres termes plus colorés ou plus littéraires, tendance légèrement plus répandue chez les Français. L'analyse nous permet de relever une ou deux occurrences : appréhender, valider, clarifier, aborder, détailler, baptiser ou en anglais consider, employ, offer, search, study, tackle. Du point de vue syntaxique, on ne note pas de réelles modifications par rapport aux structures classiques, si ce n'est l'utilisation systématique de la voix active ou passive.

On peut dire que ces fonctions confirment le rôle de présentation et d'information de l' abstract, qu'elles sont formalisées par des expressions plus ou moins figées qui relient les concepts scientifiques et que l'auteur ne peut éviter, pour preuve la liste des segments répétés en français ne contient que de telles formules. L'abstract constitue en fait un sommaire rédigé des fonctions. Ces contraintes ne semblent guère évitables puisqu'elles donnent naissance à un ensemble cohérent et bien formalisé qui contribue à rendre le message clair et facile à suivre.

\subsection{Articulation du discours}

On propose dans cette partie d'examiner les ressources linguistiques servant à exprimer les mécanismes cognitifs qui se produisent chez l'auteur au cours des différentes phases $\mathrm{du}$ raisonnement. Nous allons voir comment relier les fonctions pour construire un discours cohérent. Trois aspects ont particulièrement retenu notre attention: les marqueurs de l'articulation, les démonstratifs et les répétitions. Ce sont donc les relations entre les éléments qui constituent le discours qui nous intéressent, relations entre les mots dans la phrase et relations entre les phrases et là encore les contraintes qui surgissent.

\subsubsection{Marqueurs de l'articulation}

Le programme Alceste a répertorié tous les mots outils et locutions selon leurs fonctions. Ce qui frappe à première vue c'est le faible taux d'occurrences des mots servant à enchaîner les concepts excepté la conjonction et (127 occurrences) et and (175). Les marqueurs servant à expliciter les relations entre les phrases sont également rares : quelques marqueurs de concession, cause/effet, but ou manière, renforcement, sont peu utilisés. Seules les deux conjonctions de coordination et / ou sont fréquentes dans les deux langues. On a relevé 18 ou et 10 or. Ceci marque la relation conjonctive forte avec une fréquence élevée de et / and disjonctive faible avec ou / or. Un cas de dissociation est notoire en français : il s'agit d'une phrase contenant 49 mots avec 3 procès juxtaposés : « Une telle approche définit un cadre..., elle offre un environnement structuré, elle permet le développement de stratégies... ». Les pronoms relatifs apparaissent dans les deux langues avec 13 dont et 20 qui en français, 34 which et 37 that en tant que pronom relatif et conjonction.

Signalons une ressource spécifique à l'anglais largement exploitée dans tous les textes : la concaténation qui évite ainsi l'usage intensif de prépositions. L'exemple le plus frappant relevé comprend 11 termes: "a structured light based three dimensional, six-degree-offreedom vision system. ». Un cas de calque a été relevé en français : «Les modèles orientésobjets..." 

d'étapes et de déroulement dans le temps n'apparait pas. Les procès sont envisagés comme des notions globales et non des occurrences. La chronologie est seulement implicite. On remarque 4 textes anglais ou le prétérit marque la notion d'étape particulière du processus et 1 texte dans lequel cette notion est formalisée : «In previously reported work we have identified methods... » et la phrase suivante reprend : « These methods . .. ». Ceci est tout à fait exceptionnel.

44 En conclusion sur les interrelations marquées par les locutions et mots outils, on peut insister sur le faible taux d'occurrences et le nombre limité de formes. En règle générale les procès sont juxtaposés et les relations ne sont pas explicitées. Les structures appositives vues à propos de la fonction d'explicitation ne sont en général que des juxtapositions sans définitions des éléments :

une courbe synthétique, image des courbes brutes

a recently introduced algorithm, the marginal returns algorithm

L'analyse semble confirmer une forte tendance à mettre les procès sur le même plan sans envisager leur relation. C'est le cas pour la majorité des abstracts. Quelques cas extrêmes ont été relevés, l'un en anglais avec 64 mots et 4 phrases totalement juxtaposées, un autre avec 78 mots et 5 phrases, enfin un dernier exemple avec 121 mots et 6 phrases et un seul connecteur. En français, le texte le plus probant comprend 97 mots et 5 phrases totalement juxtaposées.

cas particulier doit être mentionné : il s'agit d'un texte anglais de 282 mots, donc plus long que la moyenne, comportant deux paragraphes, le premier dans lequel toutes les interrelations sont marquées, et le deuxième où les 8 phrases sont totalement juxtaposées.

47 A priori, il semble y avoir contradiction entre le besoin de cohérence et la juxtaposition des éléments. Nous en conclurons que l'abstract n'est probablement pas le lieu propice au développement des interrelations. Cela se fera dans l'article. Les auteurs font preuve d'une grande économie en ce qui concerne l'articulation des notions. Ils s'adressent à un public de spécialistes qui comprennent immédiatement l'enchaînement et l'ordre des idées sans la nécessité de les formaliser. On pourrait supposer de la part des Français une influence anglo-saxonne. Ceci n'est pas certain. Nous interprétons ce phénomène plutôt comme un point commun à la communauté scientifique. Nous allons voir que d'autres moyens probablement plus justifiés peuvent rendre l'ensemble cohérent. Les choix sont opérés en fonction des destinataires implicitement présents dans les esprits des auteurs lors de la rédaction.

\subsubsection{Rôle des démonstratifs}

Les démonstratifs, adjectifs et adverbes, sont nombreux et semblent avoir un rôle primordial étant donné leur nombre d'occurrences. C'est ce que nous allons essayer de démontrer. 94 occurrences en anglais, 87 en français de pronoms et adjectifs démonstratifs, les adjectifs étant plus nombreux dans cette langue, opèrent un fléchage situationnel permettant une bonne articulation du discours. Tous les textes étudiés ont exploité au mieux cette ressource; voici quelques exemples dont la liste est loin d'être exhaustive :

A new technique... is presented. This technique is based on... » et plus loin on trouve : 
This step is treated...

This motivates work...

A method... has been developed. This method utilizes...

... en se fondant sur la notion de description... . Cette description permet de...

Nous présentons les travaux entrepris... De ces travaux résulte...

Nous abordons le problème de l'estimation... Ceci est réalisé en recherchant...

49 jouent le rôle de déictiques en faisant référence aux notions qui précèdent. Des éléments autres que les démonstratifs jouent aussi ce rôle mais de façon moins importante comme tels ou such. L'avantage pour un abstract est considérable : il contribue à la cohérence tout en assurant la clarté du message en même temps que sa concision. En effet, ils rallongent peu le texte et nous savons combien cet aspect est important. Le cas de l'article défini n'a pas été considéré dans cette présentation.

\subsubsection{Répétitions}

50 souvent combinés à un adjectif démonstratif pour assurer une clarté optimum :

...On crée un polyèdre convexe... Ce polyèdre est construit à partir du polyèdre... le mot est combiné au démonstratif.

Ce papier présente une nouvelle méthode de segmentation... La méthode que nous proposons permet d'obtenir... l'ensemble des segmentations... fourni par la segmentation. Le processus de segmentation...

51 Ce texte reprend 6 fois le mot segmentation en 6 phrases. Dans un texte anglais, on note 10 occurrences de algorithme en 9 phrases. On relève peut-être l'exemple le plus caractéristique de l'intérêt de la répétition avec code vectors et coarse code book respectivement utilisés 3 et 4 fois en 4 phrases.

On imagine aisément les ambiguïtés créées par la proximité de ces deux expressions. Nous pensons que l'auteur n'avait pas le choix pour rendre son message rapidement compréhensible. Cette brève présentation semble suffire à démontrer la nécessité de répéter des mots clés. Dans un contexte aussi complexe, il n'existe pas d'autre option. C'est probablement l'exemple de contrainte le plus probant.

D'autres liens d'ordre grammatical ont été utilisés mais ils semblent moins nombreux : les adjectifs et pronoms possessifs et les pronoms personnels à la 3e personne. Il est vrai qu'ils ne suppriment pas toujours l'équivoque même pour des spécialistes. Voici probablement la raison pour laquelle les Anglophones, donnant la priorité à la clarté, optent plutôt pour la répétition de mots, encore plus que les Français.

Ces deux approches semblent traduire une des différences culturelles entre les deux peuples. Les scientifiques français attribuent encore quelque importance au style et à l'aisance de la lecture. C'est pourquoi, même dans ce contexte, ils tendent à éviter un trop grand nombre de répétitions souvent génératrices de textes lourds. Citons deux exemples où un terme global moins chargé est utilisé comme alternative à la répétition :

cette propriété permet d'analyser...

propriété fait le lien entre les deux phrases et recouvre la notion présentée dans la phrase précédente. Il en est de même dans cet exemple :

L'exploitation de cette propriété permet de définir... 
permet que nous avons déjà repéré dans la phraséologie liée à la fonction explicative a aussi un rôle articulatoire intéressant, mais c'est surtout dans la dernière partie que nous tenterons une interprétation.

Enfin, le temps du futur et du passé composé sont parfois utilisés dans l'abstract comme liens pour annoncer la chronologie à l'intérieur du texte, ce qui va suivre dans l'énoncé ou ce qui a précédé. Cette ressource est toutefois peu exploitée.

Pour conclure cette partie, on peut dire que par souci de rédiger un ensemble cohérent clair, désambiguïsé tout en restant concis, l'auteur a quelques ressources à sa disposition. Il pourra parfois opter pour l'option qu'il préfère et qui semble la plus appropriée, mais le choix reste limité et se réduit encore en fonction de la complexité et technicité. Une fois de plus, ce dernier est dicté par des contraintes fortes. Au pire, à la lumière de l'exemple cité ci-dessus, nous pouvons affirmer que le contexte impose totalement les procédés articulatoires à adopter. Ici encore les deux langues utilisent sensiblement les mêmes ressources malgré quelques préférences.

\subsection{Argumentation/énonciation} présence de l'auteur et sa relation par rapport à l'énoncé. Rappelons qu'une des contraintes est la validation du texte dans le but de le faire accepter. Ceci semble être en contradiction avec le style dépersonnalisé de présentation des faits scientifiques et l'effacement de l'énonciateur. L'enjeu peut être énorme et cette situation paradoxale montre que l'abstract est à considérer à part dans la production scientifique. Dans ce cas, quels sont les moyens mis en œuvre pour satisfaire à ces deux conditions : démontrer et convaincre?

61 L'auteur exprime son désir de s'impliquer, ceci comme moyen de vendre un produit sans a priori être perçu comme une contrainte. Certains abstracts ne présentent d'ailleurs qu'un seul repère, voire aucun. En revanche, d'autres sont plus argumentatifs. Malgré cet aspect facultatif, la notion de contrainte est pourtant présente puisque la majorité des auteurs se l'imposent. Nous n'avons relevé aucune consigne écrite ; il s'agit apparemment d'un état de fait. Nous voyons deux explications possibles : attirer l'attention et motiver puisque le lecteur va décider de l'intérêt de la recherche uniquement à partir de l'abstract, et l'influence d'un phénomène culturel très répandu, à savoir la promotion d'un produit, même dans le domaine scientifique avec toutes ses implications. C'est peut-être aussi pour cela que l'argumentation est davantage marquée dans les textes anglo-saxons. Nous citerons quelques-uns des moyens mis en œuvre.

\subsubsection{Tonalité positive}

D'après les données relevées dans les fichiers d'Alceste, la négation est peu marquée en anglais avec 13 not et insignifiante en français avec seulement 4 ne. Si la forme négative apparaît, elle est immédiatement compensée par une information positive. Le préfixe non placé devant des adjectifs de propriétés ou d'états au sens fort comme nonlinear, nonuniform, et les formes françaises calquées sur l'anglais et maintenant adoptées comme non-supervisé, non-linéaire, non-uniforme (13 occurrences en anglais et 15 en français), en un seul mot ( 7 en anglais et 2 en français) ou en 2 mots, entre toujours dans des procès positifs. La notion de doute et de restriction est compensée de la même manière. Les 
occurrences telles que peut-être, mais, pourtant, ne... que, though, nonetheless, however ne sont pas nombreuses : 13 en français et 14 en anglais, et traduisent rarement d'éléments négatifs ; sinon, elles sont suivies de termes à sens positif. Les exemples suivants prouvent la nécessité de compenser par des éléments constructifs afin de donner une impression positive :

Les ellipses sont déviantes mais compréhensibles...

Toutefois, pour résoudre les problèmes... sur un fond éclairé non uniformément, nous avons développé un algorithme efficace, facilement transposable...

Les essais conduisent à des taux d'erreurs de 5 à $10 \%$ dans les meilleurs des cas. Le but de cet article est de proposer une nouvelle approche pour la résolution de ce problème.

Though it is known that...no exact relationship ... has been given. This paper derives the exact relation...

However, even for sources that do not satisfy these conditions, the algorithm gives coders that perform close to the optimal.

However, the derivation requires the accurate determination...Fortuitously it is possible to derive...This paper proposes methods that improve the accuracy of the derivation...

...algorithm to continuously upgrade performance and has shown near $100 \%$ accuracy even after very short training periods.

Nous avons noté la même attitude à l'égard des problèmes posés ou des difficultés rencontrées. La notion de résolution intervient immédiatement. Les Anglophones semblent utiliser plus souvent cette procédure que les Français. Nous n'avons trouvé dans notre corpus aucun abstract à tonalité négative.

\subsubsection{Marqueurs de l'argumentation}

Le programme Alceste fournit la liste des occurrences des marqueurs de l'argumentation. On notera seulement les plus fréquentes. Il s'agit de avec, par (58 occurrences), pour (51) et en anglais by (39), for (118), with (46) et to. Ces embrayeurs qui mettent en relation deux termes de l'énoncé interviennent dans l'expression des fonctions et de l'articulation. Nous les avons intégrés dans cette partie bien que les trois notions soient indissociables, car leur contribution à éveiller l'intérêt du lecteur en introduisant informations et précisions est inestimable.

\subsubsection{Marqueurs de l'intensité et de la quantité}

Un autre moyen consiste à utiliser des termes qui renforcent l'assertion mais il est relativement peu employé dans l'ensemble: on a repéré 6 bien et 7 well, 4 très et 6 very. Toutefois les Anglophones n'hésitent pas à faire usage de comparatifs et superlatifs d'adjectifs d'évaluation comme greater, better, higher, lower, simpler, et en français 14 plus... ont été répertoriés. Nous n'avons pas noté d'adverbes intensificateurs anglais en ly et nous signalerons la présence exceptionnelle de considérablement et extrêmement. On ne distingue pas de différence spectaculaire entre les deux langues.

\subsubsection{Lexique argumentatif}

L'utilisation d'un lexique argumentatif est adoptée par une grande majorité d'auteurs ; on entend par là des formules servant à évaluer ou renforcer une qualité, une propriété, à émettre une appréciation, même à exprimer un point de vue pratiquement 
"émotionnel ». L'énonciateur marque son discours auquel il adhère totalement et les ressources sont nombreuses, apportant un vocabulaire riche et varié qui contraste fortement avec la phraséologie mise au service des fonctions. En voici quelques exemples :

De ces travaux résulte une nouvelle méthode...

...émet de nouvelles hypothèses et les valide...

L'expérimentation a montré la validité de cette méthode...

L'algorithme permet une coopération parfaite...

Donner une formalisation élégante...

... présentons un algorithme nettement plus simple...

Ce modèle adapté aussi bien à l'analyse qu'au traitement...

Un système... son avantage principal...

C'est un moyen rapide et élégant...

Il permet de spécifier aisément des méthodes sophistiquées.

This helps significantly reduce...

A new efficient computational technique...

Results are given to illustrate the superiority of this...

The resulting data is suitable...

This system has been successfully implemented.

...some of the authors $»$ relevant trials...

We present a computationnally efficient way of computing...

In this paper, a new approach has been proposed.... The new concept is presented...

The advantages of the technique are...

Des allusions à une amélioration par rapport au passé ou même à l'aspect innovateur sont fortement marquées surtout par les Anglophones pour insister sur l'intérêt de leurs travaux. On a relevé 8 nouveau, 2 originale, $18 \mathrm{new}$. On trouve dans le même texte : "Only a few reported successful attempts have been reported in the past....A new concept is presented. Usefulness of the method is demonstrated».

On note la marque de l'auteur à travers des mots comme prédominant, adapté, essentiel en français et en anglais delicate, appropriate, efficient, pertinent... solid(e), robust(e). Les qualités : la fiabilité, la pertinence, l'efficacité, sont mises en valeur afin de valider la recherche et la démonstration. Ce qui est intéressant, ce n'est pas le nombre d'occurrences mais la variété des termes et des stratégies.

\subsection{5. Énonciation}

D'autres moyens marquent encore la présence de l'auteur et contribuent à éveiller l'intérêt. Si quelques textes révèlent une certaine distance entre l'auteur et son énoncé, la plupart contiennent des éléments repères de l'énonciateur.

La question des pronoms a déjà été abordée. Rappelons que les Anglophones utilisent we, l'équivalent du nous français. Quelques Français ont cependant préféré le pronom indéfini on afin de garder le ton dépersonnalisé du contexte scientifique tout en évitant une surcharge de formes passives. Mais nous devons nous interroger sur le sens de ce pronom de la première personne du pluriel et voir s'il est identique dans les deux langues. Il est possible d'interpréter le nous français comme étant la traduction d'un travail qui implique plusieurs personnes autant que de la modestie. En anglais, we peut aussi inclure la relation avec le co-énonciateur, interprétation souvent justifiée en langue scientifique et aussi par la culture anglo-saxonne : l'auteur sait qu'il existe un lecteur virtuel et ceci est un moyen de l'inclure dans sa communication. Cet aspect sera repris plus loin. Après relecture, cette interprétation ne semble pas s'appliquer aux textes français. 
71 L'assertion est le mode d'énonciation prédominant en langage scientifique. Nous avons déjà remarqué le présent de l'indicatif dans les deux langues qui se présente comme une contrainte forte. Quelques emplois du futur et du passé composé ont été repérés ainsi que quelques cas de passé. L'énonciateur peut marquer son attitude vis-à-vis de son énoncé par la modalisation. Voici les modaux relevés :

6 pouvoir, 1 devoir et 4 formes de nécessité ;

22 can, 6 may, 1 must, 1 should et 5 formes de nécessité ;

5 peut-être et quelques expressions comme « it is desirable ..., it is intended that

this algorithm be implemented... , il devient possible... ».

Dans l'ensemble, les marques de la modalisation sont irrégulièrement réparties. Celle-ci peut être totalement absente. En revanche, il n'est pas rare de trouver des textes où elle apparaît plusieurs fois en quelques lignes, accompagnée par exemple en anglais du pronom we et également d'autres éléments d'argumentation. Son utilisation peut aussi traduire le niveau d'avancement des travaux du chercheur, surtout lorsque celui-ci est encore au stade de l'expérimentation. Elle est plus fréquente en anglais et ceci au moyen des verbes modaux, mais l'implication de l'auteur est souvent atténuée par l'utilisation de la forme passive. Il est vrai que les Français emploient volontiers d'autres structures modalisatrices. Notons la présence d'un texte assez différent comprenant seulement trois phrases dont voici les verbes : «La procédure... peut être améliorée. Il devient ainsi possible d'estimer... ». Cette propriété permet d'analyser...". Cette dernière phrase traduit une autre forme de modalisation. Le nombre élevé d'occurrences de telles formules comprenant permettre a attiré notre attention sur ce procédé particulièrement prisé par les Français, contrairement aux Anglophones, et ce «faire faire » ou "rendre possible » a soulevé plusieurs questions d'ordre syntaxique et sémantique. Nous n'y répondrons pas car cela nécessite une étude approfondie des contextes que nous n'avons pas encore faite. Nous voyons en cette présence distante de l'auteur une double influence, celle de la culture française où l'individu est présent et valorisé, et celle de la culture scientifique anglosaxonne qui met l'accent sur les faits scientifiques. Cette stratégie nous a semblé particulièrement intéressante à souligner pour faire ressortir une des spécificités du français.

73 Les dernières remarques à propos de la présence de l'énonciateur cherchant à valider son discours portent sur des ressources utilisées à titre exceptionnel mais intéressantes. Nous allons courir le risque d'interpréter la double stratégie mise en œuvre, même si elle n'est pas très répandue. L'auteur souhaite implicitement attirer, et maintenir dans le temps, l'attention sur ses propres travaux, actuels et futurs, tout en justifiant les limites auprès $\mathrm{du}$ lecteur. Cette "affaire à suivre » semble inévitable dans le contexte de forte et impitoyable compétition à laquelle est livrée la communauté scientifique internationale. On trouve par exemple une occurrence de : "Nous aborderons les directions futures de notre travail de recherche..." ainsi que "We view this work as a step toward the larger goal of obtaining... » qui traduisent fortement la présence de l'auteur : processus en cours, travaux précédents, aspect et chronologie marqués et forte argumentation. «A report on work in progress » qui apparaît dans un texte où la marque de l'auteur est constante.

Un auteur français a pris la liberté d'intégrer des données mathématiques appartenant à la démonstration normalement présentée dans le corps de l'article. On trouve un cas identique en anglais avec référence à une figure. Ce comportement est excessivement rare et aucune explication ne peut être envisagée pour un cas unique dans chaque langue. 
75 Le dernier point concerne la présence du co-énonciateur. Rappelons ce qui a été noté précédemment sur le pronom we qui peut traduire la relation que souhaite avoir l'auteur avec son lecteur qu'il englobe. Dans un domaine où l'impersonnel, le formel et les fonctions cognitives sont prédominants, n'est-ce pas une façon d'intégrer, outre quelques termes, un autre registre appartenant au domaine de l'affectivité? Il est difficile de justifier cette interprétation et de confirmer ce sens dans les abstracts étudiés. Quelques expressions faisant allusion au public scientifique rappellent également que des lecteurs virtuels existent: «Différentes techniques bien connues... . It is known that... . With known misalignment... »

En conclusion, on peut dire que l'argumentation fait partie inhérente de l'abstract d'après la fréquence des repères. Les ressources ont des occurrences quelquefois peu nombreuses mais elles sont variées et exploitées sous de multiples formes, ce qui surprend parfois le lecteur non scientifique. Sans exagérer, on irait jusqu'à dire que chaque auteur a adopté un traitement propre. Donc ce qui semble synonyme de liberté est tout de même dicté par une même loi qui est celle de valider un énoncé pour motiver un lecteur virtuel. L'auteur peut à la fois assumer son texte et marquer une certaine distance. Malgré l'existence dans les deux langues de formes extrêmes, textes d'auteurs ou au contraire totalement impersonnels, de l'ordre de un ou deux, le traitement de l'argumentation n'est nullement négligé, avec quelques différences. Globalement nous pouvons en conclure que l'anglais semble opter pour la grammaticalisation alors que le français privilégie l'aspect lexical. Ce point ne sera pas développé ici mais il mériterait d'être exploré afin de mieux percevoir les différences culturelles.

77 Mais il reste vrai qu'au même titre que la démonstration, sa validation représente, d'après le corpus étudié et les données quantitatives, une obligation pour l'auteur, non dite ni imposée par qui que ce soit mais implicitement indispensable. Un rapide coup d'œil sur les corpus de mathématiques et mécanique semblent confirmer, peut-être de manière légèrement moins marquée, cet état de choses mais les analyses quantitatives n'étant pas faites, il est difficile d'en évaluer l'importance et d'établir des similitudes.

\section{Conclusion}

De l'analyse des abstracts on peut tirer les conclusions suivantes. Les contraintes sont importantes et on ne peut pas nier que l'informaticien qui doit réduire son article en un abstract se trouve relativement coincé. Il doit respecter à la fois les consignes éventuelles de l'éditeur et les règles de l'abstract en matière de contenu et de style. Quels que soient le degré de technicité et la complexité de ses recherches, il devra être clair et intéressant. N'étant pas spécialistes scientifiques, nous ne sommes pas en mesure d'en juger. Nous pouvons toutefois constater les moyens mis en œuvre pour expliciter et désambiguïser d'une part, motiver et argumenter pour valider d'autre part. En revanche, la concision n'est pas toujours de mise puisque la longueur et l'importance du développement varient sensiblement.

79 S'il n'a pas reçu de consignes rigoureuses et précises concernant l'usage de la langue, l'auteur a une certaine latitude, c'est-à-dire qu'il peut exploiter toutes les ressources de la langue, à l'intérieur de ce cadre dont nous avons parlé, connaissant les impératifs du message. 
80 Il est difficile de parler de forme canonique pour l'abstract, du moins en informatique, mais plutôt de constantes que l'on retrouve, à part quelques exceptions, dans tous les textes :

- la phraséologie dont certains éléments ne seraient pas indispensables, l'utilisation de mots « joker » dans un contexte spécialisé et précis ;

- l'absence de chronologie ;

- le mode d'articulation pour la démonstration ;

- des structures passives ou actives avec sujets inanimés de verbes animés ;

- la modalisation peu ou irrégulièrement marquée ;

- la présence peu marquée du co-énociateur ;

- le poids de l'argumentation ;

- l'approche assez similaire dans les deux langues malgré quelques influences culturelles.

81 La liberté est somme toute très relative; nous avons examiné un certain nombre de paramètres qui peuvent déterminer les choix linguistiques. Enfin, nous avons constaté que certains éditeurs, réviseurs ou responsables de publications jugent aussi selon leurs goûts personnels et laissent plus ou moins de latitude. Quelques auteurs seulement font preuve d'un style personnel, soit par le biais de la modalisation, des formules argumentatoires, de la qualité du développement soit au contraire par l'absence de détails, la dépersonnalisation totale et la juxtaposition des procès. Clôturons cet exposé en mentionnant deux extraits de notes d'éditeurs :

L'abstract est la partie la plus importante. Il reprend les termes du titre, présente le sujet, les idées principales et résume la discussion qui va suivre. C'est une table des matières en prose. Il doit être concis, clair et ne laisser aucune ambiguïté. L'abstract permet au lecteur de décider rapidement s'il va continuer la lecture. Les lecteurs sont très occupés et doivent voir immédiatement si l'article est intéressant. Un abstract bien fait est encourageant.

Puisque l'information de nos jours est fondée sur le titre et l'abstract, ces derniers doivent être rédigés avec le plus grand soin. Certains auteurs trouvent l'abstract difficile, il leur est conseillé de se faire aider en cas de besoin.

Nous vérifions ainsi quelques-unes des constatations relevées au cours de l'analyse, ce qui était d'ailleurs déjà annoncé au niveau du titre. On peut se demander s'il n'y a pas actuellement une sorte de consensus qui incitent les auteurs à s'imposer eux-mêmes leurs règles. Cette situation est de par ce fait sujette à évolution comme cela a déjà été signalé, et les règles en vigueur actuellement, qui ont peut-être déjà changé, peuvent très bien être modifiées au fur et à mesure par quelques auteurs reconnus qui feront autorité et s'octroieront la liberté de mettre en place une nouvelle règle du jeu, donnant naissance à de nouvelles contraintes, mais ceci est un autre débat.

\section{BIBLIOGRAPHIE}

Guillemin-Flescher, Jacqueline. 1981. Syntaxe comparée du français et de l'anglais. Problèmes de traduction. Gap : Ophrys. 
Kocourek, Rostislav. 1982. «La langue française de la technique et de la science ». La Documentation française. Wiesbaden, Allemagne : Oscar Brandstetter Verlag.

\section{RÉSUMÉS}

L'objet de l'abstract est de présenter en quelques lignes les points essentiels d'un article. Les contraintes extérieures et les exigences inhérentes à cette forme de discours déterminent le langage utilisé par l'auteur. Nous proposons une analyse comparative du français et de l'anglais à partir d'un corpus d'abstracts en informatique traité par le programme Alceste. Les résultats permettent de dégager le style langagier, les attitudes différentielles entre Anglophones et Français et la marge de liberté de l'auteur.

The abstract aims to briefly introduce the major ideas of a paper. The language used is determined by external constraints and requirements inherent in this form of discourse. We propose a corpus-based comparative analysis of French and English abstracts in computer science processed by Alceste software. The results bring to light language style, differential attitudes between French and native speakers of English, and the author's margin of freedom.

\section{INDEX}

Keywords : abstract, discourse, stylistic analysis

Mots-clés : analyse stylistique, discours, style

\section{AUTEUR}

\section{ELIZABETH CROSNIER}

CCG - INSA Toulouse. elizabeth.crosnier@cict.fr 